\title{
Radiance Measurement for Low Density Mars Entry
}

\author{
Brett A. Cruden ${ }^{1}$ \\ ERC Inc., Moffett Field, CA 94035
}

\begin{abstract}
We report measurements of radiance behind a shock wave in Martian simulant $(96 \%$ $\mathrm{CO}_{2}, 4 \% \mathrm{~N}_{2}$ ) atmosphere at conditions relevant for aerodynamic decelerators. Shock waves are generated in the NASA Ames Electric Arc Shock Tube (EAST) facility at velocities from 6-8 $\mathrm{km} / \mathrm{s}$ and freestream densities from $1.2-5.9 \times 10^{-4} \mathrm{~kg} / \mathrm{m}^{3}(0.05-0.25$ Torr, corresponding to $35-50 \mathrm{~km}$ altitude). Absolute radiance is measured as a function of wavelength and position in the shock. Radiance measurements extend from the vacuum ultraviolet to near infrared $(120-1650 \mathrm{~nm})$. As at higher density/velocity, radiation is dominate by $\mathrm{CO} 4^{\text {th }}$ positive radiation in the vacuum ultraviolet, though $\mathrm{CN}$ contribution is also significant. At most low density conditions, the shock does not relax to equilibrium over several centimeters. A small number of measurements in the mid-infrared were performed to quantify radiation from the fundamental vibrational transition in $\mathrm{CO}$, and this is found to be a minor contributor to the overall radiance at these speeds. Efforts to extend test time and reliability in the $60 \mathrm{~cm}(24 ")$ shock tube will be discussed in the full paper.
\end{abstract}

\section{Introduction}

$\mathrm{N}$ EW concepts for landing high mass vehicles on the Martian surface have recently led NASA to invest in developing large cross section aerodynamic decelerator technologies. These approaches decelerate vehicles higher in the atmosphere than have been done traditionally for ballistic Martian entry. From an aerothermal perspective, this requires characterization of a new regime for atmospheric entry. Low density shocked gas takes longer to approach an equilibrium condition and therefore the shock chemistry will be determined by $\mathrm{CO}_{2} / \mathrm{N}_{2}$ kinetics. Large diameter decelerators yield greater shock stand-off distances, which increases the volume of radiating gas. Therefore, though radiation may be decreased due to lower density on a volumetric basis, the areal radiative heating received by the entry vehicle may increase. Additionally, the larger magnitude expected for nonequilibrium radiation may make radiation of greater concern than in ballistic entry problems. Non-equilibrium models for shock-layer radiation apply many simplifying approximations and as such carry significant uncertainty. To address these issues, we have undertaken to measure radiative profiles through shocks travelling at conditions relevant for Martian aerocapture.

In previous work, we have used the Ames Electric Arc Shock Tube to measure shock-layer radiation at a variety of conditions relevant for entry to Earth, Mars and Venus.[1-3] These conditions have generally involved pressures and velocities that exceed those expected for Aerocapture trajectories. The present work extends these conditions to lower pressures and velocities that are consistent with trajectories used by the Mars Aerocapture System Study (MASS)[4] and Hypersonic Inflatable Aerodynamic Decelerator (HIAD)[5]. These conditions span velocities from 6-8 km/s and altitudes of 35-50 km (0.05-0.25 Torr, 1.1-5.9 x $\left.10^{-4} \mathrm{~kg} / \mathrm{m}^{3}\right)$. Data is reported with spectral and spatial resolution for wavelengths spanning from the Vacuum Ultraviolet to Mid-Infrared.

\section{Experimental}

The Ames Electric Arc Shock Tube has been described in detail in previous publications.[2] In brief, it consists of a 4" $(10.16 \mathrm{~cm})$ inner diameter aluminum tube coupled to an electric arc driver source and dump tank, with the three sections being isolated with aluminum diaphragms. Approximately $7.5 \mathrm{~m}$ downstream of the driver diaphragm are four spectrometers with optics focusing on a $12.5 \mathrm{~cm}$ wide section of the tube. The four sets of optics image identical axial positions, but at different azimuthal angles, passing through the centerline of the shock tube. Optics are enclosed in vacuum to allow imaging from the vacuum ultraviolet through the mid-IR. The driver section consists of a 1.3L conical section pressurized with $100 \mathrm{psi}$ of He. To initiate a shock within the tube, an arc

\footnotetext{
${ }^{1}$ Sr. Research Scientist, Aerothermodynamics Branch, NASA Ames Research Center, MS 230-3, Moffett Field, CA, AIAA Sr. Member.
} 
discharge is created within the driver section at voltages between $6.8-11 \mathrm{kV}(9-30 \mathrm{kV}$ with buffer) from a $863 \mu \mathrm{F}$ capacitor bank. The resulting rise in pressure and temperature within the driver ruptures the upstream diaphragm and induces a shock wave traveling down the tube. The passage of the shock is monitored via piezoelectric shock sensors to produce an $\mathrm{x}$-t diagram, differentiation of which provides a measurement of velocity. When the shock reaches the test section, CCD arrays attached to the spectrometers are gated to obtain an image of the shock wave. The spectrometers are calibrated using absolute radiance sources - a 16", $1.2 \mathrm{~kW}$ integrating sphere (Spheroptics) in the visible through near infrared, a $1200 \mathrm{C}$ blackbody in the mid-infrared and a $30 \mathrm{~W} \mathrm{D}_{2}$ lamp (McPherson) in the vacuum ultraviolet and ultraviolet. The $\mathrm{D}_{2}$ lamp calibration is anchored to the integrating sphere calibration at an overlap region between $300-360 \mathrm{~nm}$.

Testing at low density presents certain challenges, particularly the ability to obtain reliable test time in the shock wave. Due to the low density of the driven shock, asperities in the driver shock may not planarize by the time the shock reaches the test section, resulting in ill-defined shock conditions. To address this issue at high velocity in previous work, we had employed a buffer section between the driver and driven gas which helped to planarize the contact surface prior to interaction with the test gas.[3] At low density, this approach proved less successful. Due to the lower temperature of the buffer gas, radiation is not observed in this region, and with the decaying non-equilibrium radiation profile observed in these conditions, it was difficult to ascertain where mixing of the cold buffer and driven gas occurred. In the end, more definitive results were obtained without using the buffer, although success rates from $6-7.5 \mathrm{~km} / \mathrm{s}$ and $0.05-0.1$ Torr were between 40-50\%. Current work applying the EAST 24" tube to address this low success rate will be reported in the full paper. Conditions studied in this work are shown in Fig. 1. Also shown are sample trajectories from MASS and HIAD, adjusted to a density-equivalent pressure at laboratory conditions.

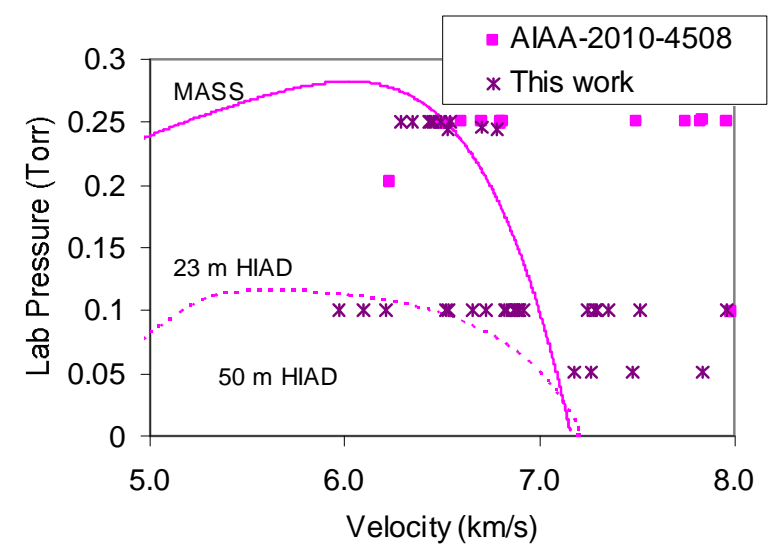

Figure 1. Conditions studied in this work. Also shown are sample trajectories for Aerodynamic Decelerators and conditions studied in Ref. [1].

\section{Results}

An example of data obtained is shown in Fig. 2 for a nominal test case of $7.5 \mathrm{~km} / \mathrm{s}$ and 0.05 Torr. The 3D image shows volumetric spectral radiance as a function of position (y-axis) and wavelength (x-axis). The region shown corresponds primarily to $\mathrm{CN}$ violet radiation. Integration of the volumetric spectral radiance versus wavelength yields the volumetric radiance as displayed on the vertical $2 \mathrm{D}$ plot. The shock front is observed at a position of approximately $3 \mathrm{~cm}$, at which point the radiance rises to a maximum and then decays monotonically to approximately $6 \mathrm{~cm}$ where the contract front is. The presence of the contact front is characterized by the appearance of multiple new lines corresponding to aluminum and tungsten contamination from the driver section. The valid test region lies between these two points. The horizontal plot shows an average cross-section in this region, as denoted by the horizontal red lines. Peaks at 359, 388, 422 are characteristic band heads of the CN Violet system, while the band at $474 \mathrm{~nm}$ is attributable to the $\mathrm{C}_{2}$ Swan system. The shape of the decay shows a much more gradual decay than in reference [1], indicating a much slower approach to equilibrium than observed at higher density condition. Full decay to equilibrium is not observed within the test time at the two lower pressure 
conditions studied ( 0.1 and 0.05 Torr), while equilibrium is achieved within a few centimeters at higher pressure (0.25 Torr) condition.

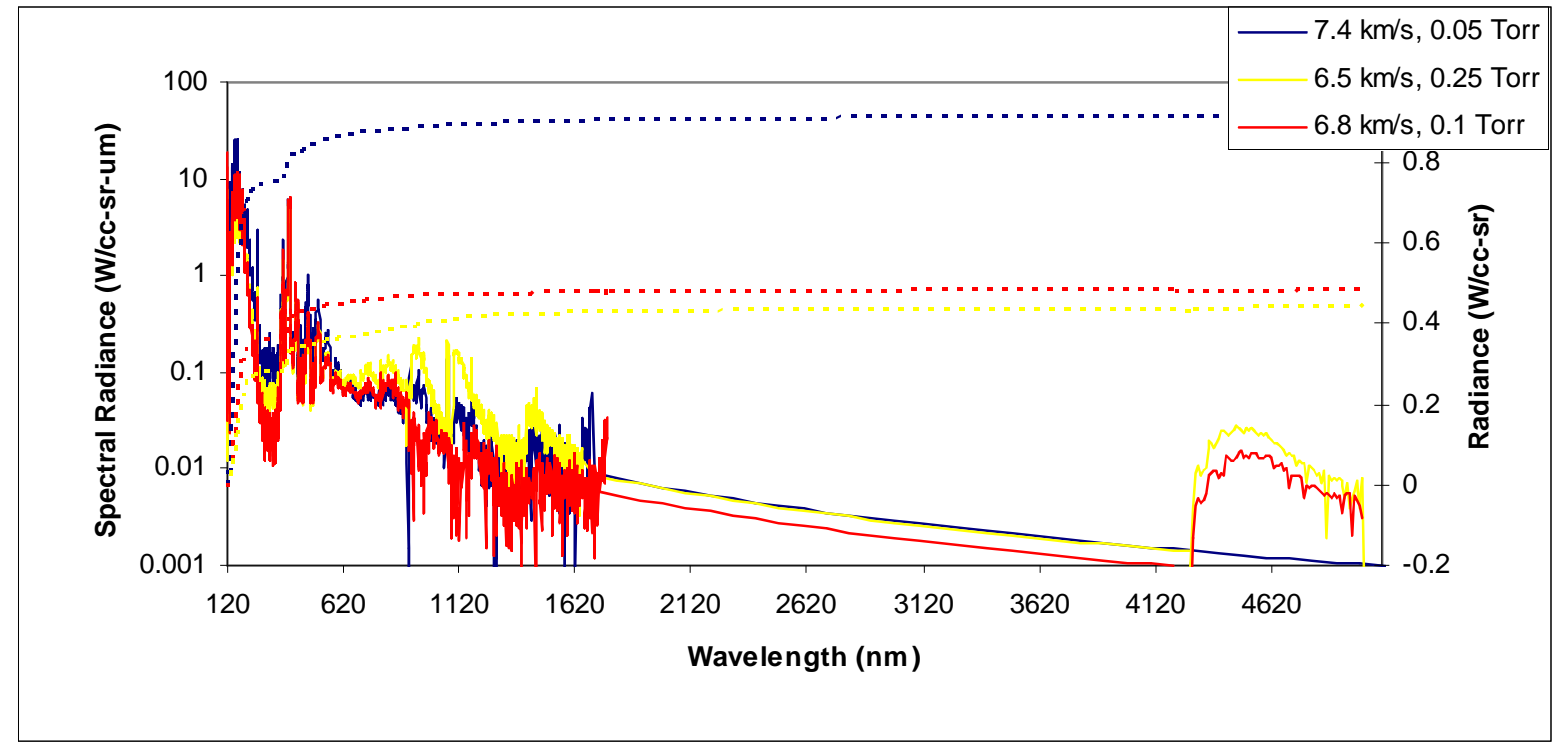

Figure 3. Radiance behind the shock for three separate test cases.

Fig. 3 shows a spectral cross-section collected just behind the shock front (i.e. as shown in Fig. 2) for three conditions corresponding to the three pressure conditions studied. Velocity is increased at lower pressure conditions as might be expected on an entry trajectory. For these three plots, in non-equilibrium the reduction of density is approximately balanced by an increase in velocity and all conditions have similar radiance magnitude. Integrated spectral radiance is shown as the dotted lines and plotted on the second y-axis. The $7.4 \mathrm{~km} / \mathrm{s}$ condition has approximately a factor of 2 larger radiance than the slower conditions, and it is apparent from the integral that most of the difference arises in the vacuum ultraviolet region where $\mathrm{CO} 4{ }^{\text {th }}$ Positive radiation is dominant. The magnitude of $\mathrm{CN}$ radiation is similar for most cases. Beyond $1650 \mathrm{~nm}$, the spectra are extrapolated assuming a $\lambda^{-2}$ dependence, as in Kramers-Unsold continua formula. For two of the cases shown, additional data was collected from 4000-5000 $\mathrm{nm}$ and shows a band structure which differs from that reported in [1]. This region is attributable to the fundamental vibrational transition of $\mathrm{CO}$. Due to dissociation at elevated temperature, the $\mathrm{CO}_{2}$ density is too low in this region to be observed. In terms of the integration of spectral radiance, this region contributes relatively little to the overall radiance, suggesting it is safe to neglect this part of the spectrum under these conditions.

Since equilibrium is not attained in these shocks, an alternative metric is applied to assess the level of nonequilibrium radiation as a function of velocity. To do this, the radiance over different spectral regions is integrated from $2 \mathrm{~cm}$ in front of and $2 \mathrm{~cm}$ behind the peak radiance. This integration should capture the non-equilibrium peak, is long enough to contain the camera smearing function, and is sufficiently short as to not be contaminated by the contact front. This integration is plotted in Fig. 4 as a function of velocity for two of the pressure conditions and shown to display an almost linear relationship with velocity in this range. Also shown for comparison is the identical analysis of data reported in Ref. [1], and good quantitative agreement is obtained.

\section{Conclusions}

The final paper will have detailed conclusions of the study, including the results of initial characterization in the 24 " shock tube. The paper will also provide a first order estimate of radiative heating and impact during Martian entry for HIAD-like entry vehicles.

\section{Acknowledgments}

The author would like to acknowledge Mark McGlaughlin, James Joyce and Ramon Martinez for operation of the EAST Facility. This work supported by the Fundamental Aeronautics Program, Hypersonic Project, Aerodynamics/Aerothermodynamics/Plasmadynamics discipline, Dr. Deepak Bose, Project Manager. Dr. Cruden is supported by NASA prime contract NNA10DE12C to ERC Inc. 

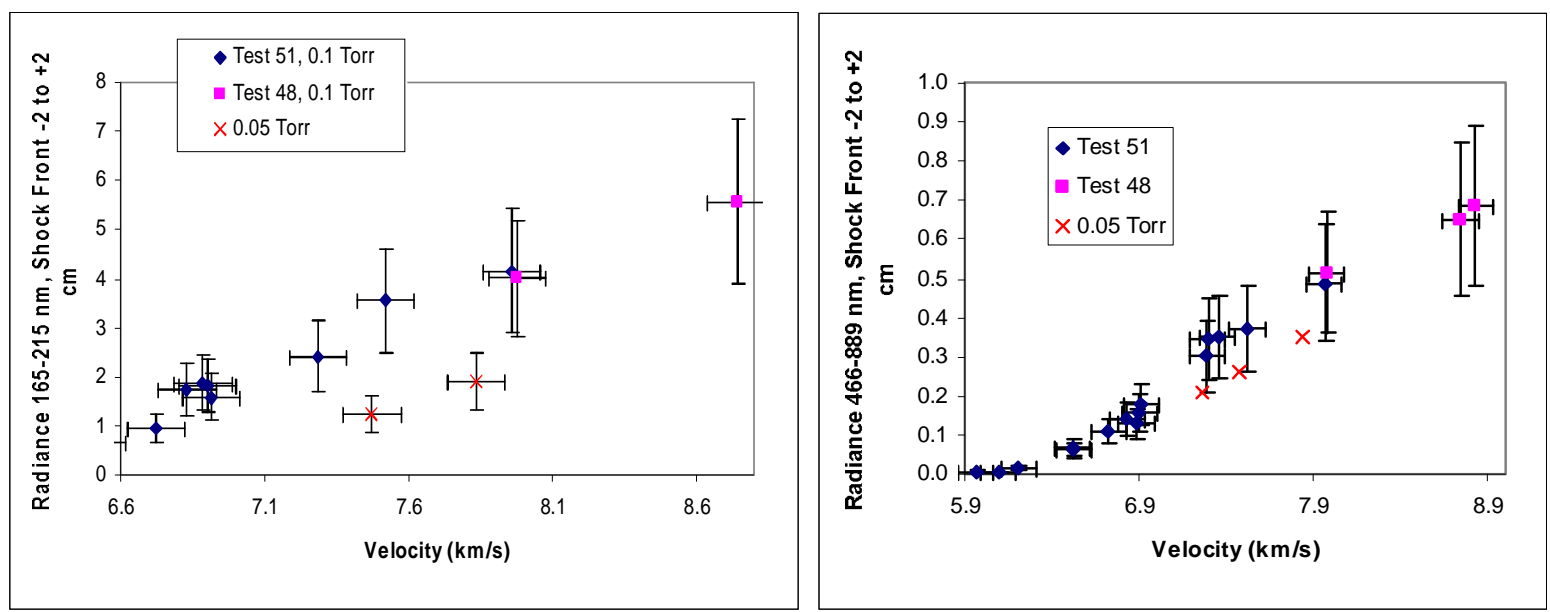

Figure 4. Non-equilibrium radiance as a function of velocity obtained in two different spectral regions

\section{References}

${ }^{1}$ Cruden, B. A., Prabhu, D., Martinez, R., Le, H., Grinstead, J. H., and Bose, D. "Absolute Radiation Measurement in High Mass Venus and Mars Entry Conditions," 10th AIAA/ASME Joint Thermophysics and Heat Transfer Conference. Chicago, IL, 2010, pp. AIAA-2010-4508.

${ }^{2}$ Cruden, B. A., Martinez, R., Grinstead, J. H., and Olejniczak, J. "Simultaneous Vacuum Ultraviolet through Near IR Absolute Radiation Measurement with Spatiotemporal Resolution in an Electric Arc Shock Tube," 41 st AIAA Thermophysics Conference. San Antonio, TX, 2009, pp. AIAA 2009-4240.

${ }^{3}$ Cruden, B., Le, H., and Martinez, R. "Electron Density Measurement in Re-entry Shocks for Lunar Return," 42nd AIAA Thermophysics Conference. Honolulu, HI, 2011, pp. AIAA 2011-3628.

${ }^{4}$ Wright, H. S., Oh, D. Y., Westhelle, C. H., Fisher, J. L., Dyke, R. E., Edquist, K. T., Brown, J. L., Justh, H. L., and Munk, M. M. "Mars Aerocapture Systems Study." NASA, 2006.

${ }^{5}$ Dwyer-Cianciolo, A. M., Davis, J. L., Komar, D. R., Munk, M. M., Samareh, J. A., Powell, R. W., Shidner, J. D., Stanley, D. O., Wilhite, A. W., and Kinney, D. J. "Entry, Descent and Landing Systems Analysis Study: Phase 1 Report," NASA Technical Memorandum TM-2010-216720, pgs, pp. 71-79. 